\title{
WEIGHTED EMPIRICAL MODE DECOMPOSITION FOR PROCESSING GNSS POSITION TIME SERIES WITH THE CONSIDERATION OF FORMAL ERRORS
}

\author{
Xiaomeng QIU ${ }^{1)}$, Fengwei WANG ${ }^{2)}$ *, Yunqi ZHOU ${ }^{3)}$ and Shijian ZHOU ${ }^{4)}$ \\ ${ }^{1)}$ Yangtze River College, East China University of Technology, Fuzhou, China \\ ${ }^{2)}$ State Key laboratory of Marine Geology, Tongji University, Shanghai, China \\ ${ }^{3)}$ School of Geographical Sciences, University of Bristol, Bristol, United Kingdom \\ ${ }^{4)}$ Nanchang Hangkong University, Nanchang, China \\ *Corresponding author's e-mail: wangfw-foster@tongji.edu.cn
}

\begin{tabular}{l} 
ARTICLE INFO \\
\hline Article history: \\
Received 23 March 2021 \\
Accepted 22 July 2021 \\
Available online 31 July 2021 \\
\hline
\end{tabular}

Keywords:

GNSS Position Time Series

Empirical Mode Decomposition

Formal Errors

Signal Extraction

Weighted Empirical Mode Decomposition

\begin{abstract}
Empirical Mode Decomposition (EMD) is suitable to process the nonlinear and non-stationary time series for filtering noise out to extract the signals. The formal errors are provided along with Global Navigation Satellite System (GNSS) position time series, however, not being considered by the traditional EMD. In this contribution, we proposed a modified approach that called weighted Empirical Mode Decomposition (weighted EMD) to extract signals from GNSS position time series, by constructing the weight factors based on the formal errors. The position time series over the period from 2011 to 2018 of six permanent stations (SCBZ, SCJU, SCMN, HLFY, FJPT, SNXY) were analyzed by weighted EMD, as well as the traditional EMD. The results show that weighted EMD can extract more signals than traditional EMD from original GNSS position time series. Additionally, the fitting errors were reduced $14.52 \%, 12.25 \%$ and $8.06 \%$ for North, East and Up components for weighted EMD relative to traditional EMD, respectively. Moreover, 100 simulations of four stations are further carried out to validate the performances of weighted EMD and traditional EMD. The mean Root Mean Squared Errors (RMSEs) are reduced from traditional EMD to weighted EMD with the reductions of $9.08 \%, 9.63 \%$ and $6.84 \%$ for East, North and Up components, respectively, which highlights the necessity of considering the formal errors. Therefore, it reasonable to conclude that weighted EMD can extract the signals more than traditional EMD, which can be suggested to analyze GNSS position time series with formal errors.
\end{abstract}

\section{INTRODUCTION}

The GNSS position time series can provide valuable basic data for Geodesy and Geodynamics research. Therefore, position time series have been used in nonlinear change, crustal deformation, post-glacial rebound and geocentric movement widely (Zhu et al, 2017; Li et al., 2020; Peltier et al., 2015; Tobita et al., 2016). Moreover, position time series not only reflect the linear tectonic movement of permanent stations, but also reveal the nonlinear movement of reference stations due to various geophysical phenomena. It is very important to study the nonlinear variation characteristics of position time series for establishing and maintaining dynamic Eearth reference frame.

Due to the influence of the external environment, the error of observation technology and reference frame of the station, various signals and noise are included in GNSS position time series (Bogusz and Klos, 2016; Chen et al., 2020), such as trend term, seasonal signals, offsets and noise (Qian et al., 2015; Ming et al., 2016). A linear trend, annual and semiannual oscillations, and offsets can be estimated from GNSS position time series by using suitable method (Nikolaidis, 2002). The method of maximum likelihood estimation of Create and Analyze Time Series (CATS) software was normally used to analyze the noise characteristic of position time series, which obtained the type of noise at North, East and Up components are not consistent (Huang et al., 2014). It is found that different complicated noise models would influence station's linear velocity and its uncertainty with maximum discrepancy larger than $1 \mathrm{~mm} / \mathrm{a}$ ( $\mathrm{Li}$ et al., 2012). The effective separation of noise and signal has a great influence on obtaining accurate noise variance and determining the uncertainty of linear and non-linear motion parameters estimation (Ma, 2019). Therefore, it is very important to choose an appropriate signal extraction method.

Several studies have been carried out to extract signals from GNSS position time series, such as Wavelet Analysis (WA), Singular Value Decomposition (SVD), Singular Spectrum Analysis (SSA), etc. (Wei et al, 2019; Ma et al., 2020a; Ma et al., 2020b; Dai et al., 2021; Tufts et al., 1982). However, these approaches have some limitations to deal with the nonlinear time series. The denoising performance of WA is easily affected by wavelet basis, decomposition level and threshold, what's more, the useful information will be eliminated in the 
process of threshold processing (Song et al., 2017). The key issue of SVD is to determine the reconstructed order of singular values for reconstructing signal accurately. Normally, it is difficult to determine the reconstructed order especially when the singular value difference spectrum criterion is used to handle the time series with strong trend (Liu and Chen, 2019; Lei and Zhong, 2013). Similarly, the window length and reconstructed order are two key parameters for SSA to process time series. If the parameters are not selected accurately that some signals will be overwhelmed or part of noise will be treated as signal (Golyandina and Zhigljavsky, 2013; Dai et al., 2021). Huang et al. (1998) first proposed an adaptive time-frequency analysis approach named Empirical Mode Decomposition (EMD). Zhang et al. (2018) successfully applied EMD to separate signal and noise from the original time series, and extracted the periodic signals from GNSS time series accurately.

The traditional EMD processes the GNSS time series without taking the formal errors into account, which assuming that all epochs of the position time series with equal weight for different epochs. This is unreasonable because the precision of GNSS position time series at different epoch are varied ( $\mathrm{Li}$ et al., 2015; Wang et al., 2018). Therefore, the formal errors should be considered when using EMD to extract signals from GNSS position time series. Li et al. (2015, 2018) considered the formal errors in spatiotemporal filtering by using Principal Component Analysis (PCA) to extract common mode error from regional GNSS position time series. Ji et al. (2020) proposed weighted wavelet analysis to extract signals from GNSS position time series, in which the weight factors are constructed based on the formal errors. To the best of our knowledge, almost all EMD approaches did not take the formal errors into account when analyzing the GNSS position time series. Therefore, we propose a modified EMD named weighted EMD.

The rest of this paper is organized as follows: the methodology of traditional EMD and weighted EMD are presented in Section 2. Signal extraction from real position time series of six stations from the Crustal Movement Observation Network of China (CMONOC) during the period from 2011 to 2018 is analyzed in Section 3. Synthetic time series analysis is carried out in Section 4. Conclusions are given in Section 5.

\section{METHODOLOGY}

\subsection{TRADITIONAL EMPIRICAL MODE DECOMPOSITION}

EMD is an adaptive time-frequency analysis approach which has advantages in dealing with nonlinear and non-stationary time series. The specific methodology of traditional EMD is as follows:

The position time series $x(t)$ can be decomposed into several Intrinsic Mode Function (IMF) components with frequency from high to low and a residual term by using EMD (Huang et al., 1998).
Noise reduction can be realized by reconstructing the residual term and some low-frequency IMF components. Generally, high frequency IMF components are mainly related to noise information, while low frequency IMF components and residual term contain characteristic information and trend of the time series, being recognized as signals. For EMD, two conditions should be satisfied with each IMF component (Huang et al., 1998):

(1) In the whole sequence, the number of extrema and the number of zero crossings must either equal or differ at most by one;

(2) All the local maxima are connected by a cubic spline line as the upper envelope. Repeat the procedure for the local minima to produce the lower envelope. The mean value of upper envelope and lower envelope is zero. However, IMF components are difficult to strictly meet the second condition in the actual time series decomposition process. The threshold expression for stopping filtering of each IMF component is expressed as:

$\mathrm{SD}=\sum_{t=1}^{N}\left[\frac{\left|h_{k-1}(t)-h_{k}(t)\right|^{2}}{h_{k-1}^{2}(t)}\right]$

where $h_{k-1}(t)$ and $h_{k}(t)$ are two adjacent data sequences in the screening process of each IMF component, $\mathrm{N}$ denotes the length of time series. SD represents the threshold for each IMF component to stop filtering, which is usually taken as $0.2-0.3$ (Huang et al., 1998).

The steps of traditional EMD for processing the position time series $x(t)$ are presented as follows:

(1) All the maxima points and minima points are extracted in original position time series, and then upper envelope and lower envelope are obtained by using cubic spline interpolation, which is generated mean sequence $m_{1}(t)$ by their average value. Differences between the original position time series and mean sequence can be expressed as

$h_{1}(t)=x(t)-m_{1}(t)$

(2) Repeat $k$ times of step (1), if $h_{k}(t)$ satisfied the conditions of IMF component, which considered as the first IMF component $\left(\mathrm{IMF}_{1}\right)$.

(3) The first IMF component is subtracted from original position time series to generate a new data series,

$x_{2}(t)=x(t)-I M F_{1}(t)$

(4) Take $x_{2}(t)$ as original data series and repeat the above steps to obtain $m$ IMF components and a residual term only when the residual term satisfies the monotonic condition. Therefore, the original data series can be expressed as:

$x(t)=\sum_{i=1}^{m} I M F_{i}+r(t)$

where $m$ and $i$ denote the number of IMF component and the index of IMF component, $r(t)$ represents the residual term. 
For traditional EMD, the boundary IMF component between signal and noise should be determined. Generally, the signal and noise are separated by using correlation coefficient criterion, by which the IMF component with minimum correlation coefficient for the first time is the boundary IMF component. The correlation coefficient between simple IMF component and original time series is computed as follows (Zhang et al., 2018)

$R(x, I M F)=\frac{\sum_{t=1}^{N}(x(t)-\bar{x})\left(I M F_{i}(t)-\overline{I M F_{i}}\right)}{\sqrt{\sum_{t=1}^{N}(x(t)-\bar{x})^{2}} \sqrt{\sum_{t=1}^{N}\left(I M F_{i}(t)-\overline{I M F_{i}}\right)^{2}}}$

where $x(t)$ and $I M F_{i}(t)$ represent the original time series and the $i$-th IMF component; $\bar{x}=\frac{1}{N} \sum_{t=1}^{N} x(t)$; $\overline{I M F}_{i}=\frac{1}{N} \sum_{t=1}^{N} I M F_{i}(t)$.

The boundary IMF component is incorporated into noise, the low frequency IMF components after the boundary IMF component and residual term are used to reconstructed the signal as follows:

$\hat{x}(t)=\sum_{k=K+1}^{m} I M F_{k}+r(t)$

where $\hat{x}(t)$ is the reconstructed signal; $K$ is the index of boundary IMF component. In other words, the IMF components before index $K$ are recognized as noise.

\subsection{WEIGHTED EMPIRICAL MODE DECOMPOSITION BY CONSIDERING THE FORMAL ERRORS}

For traditional EMD, the Equations (1) (6) are usually applied to analyze the GNSS position time series without taking formal errors into account, which assuming that all epochs of observations have equal weights. Normally, the position time series $x(t)(t=$ $1,2, \cdots, N)$ can be expressed by the signal $s(t)$ and the noise $e(t)$ as follows

$x(t)=s(t)+e(t)(t=1,2, \cdots, N)$

Considering that the formal errors derived from daily GNSS solutions are varied for different epochs due to the different surrounding. Consequently, it is unreasonable to directly treat all epochs of observations with unit weight when processing the position time series. Similar to Li et al. (2018), we just weight the noise term mainly due to that the formal errors are related to the noise (Dong et al., 2006), the generated time series is expressed as follows

$x^{\prime}(t)=s(t)+\frac{\sigma_{0}}{\sigma(t)} e(t) \quad(t=1,2, \cdots, N)$

where $x^{\prime}(t)$ represents the generated time series, $\sigma_{0}$ is a constant denoting the standard deviation of unit weight of the formal errors, $\sigma(t)$ denote the formal error of the original time series at $t$-th epoch. According to the definition of weight, we have

$p(t)=\frac{\sigma_{0}^{2}}{\sigma^{2}(t)}$ $p(1) \sigma^{2}(1)=p(2) \sigma^{2}(2)=\cdots=p(N) \sigma^{2}(N)=\sigma_{0}^{2}$

Therefore, processing the generated time series with traditional EMD is equivalent to processing the original time series by introducing a weight factor. In order to keep the total energy of the generated time series unchanged, the sum of weights for all epochs should be equal to the number of available epochs ( $\mathrm{Li}$ and Shen, 2018; Ji et al., 2020; Dong et al., 2006; Shen et al., 2018), which can be expressed as follows

$\sum_{t=1}^{N} p(t)=N$

According to Equations (10) and (11), the standard deviation of unit weight $\sigma_{0}$ can be derived as

$\sigma_{0}=\sqrt{N / \sum_{t=1}^{N} \frac{1}{\sigma^{2}(t)}}$

Once $\sigma_{0}$ is determined, it is easy to obtain the weight factors $\sigma_{0} / \sigma(t)$ by Equation (9). Firstly, the traditional EMD is used to obtain the initial signal and noise. Secondly, we can update $e(t)$ by subtracting $s(t)$ from Equation (7) and form the generated time series with Equation (8). Lastly, the generated time series is iteratively processed by EMD to extract signal and noise, and compared with traditional EMD. Considering that with the number of iterations increases, the accuracy of the extracted signals is not improved much, but iterative procedure needs more time. Therefore, the number of iterations is equal to 2 in this study. The flow of weighted EMD is shown in Figure 1.

\section{REAL GNSS POSITION TIME SERIES ANALYSIS}

To verify the performance of weighted EMD, the position time series of six permanent stations of CMONOC were analyzed with the comparison of traditional EMD and their corresponding locations are presented in Figure 2. There are few missing data at these stations. There are three stations (SCBZ, SCJU, SCMN) in the Sichuan province, while the others (HLPY, FJPT, SNXY) in different provinces. Since the observations of part stations started in July 2010, the time span was from 2011 till 2018 in this study. Together with the position time series, formal errors were downloaded from the GNSS data products of China earthquake administration, which the website is http://www.cgps.ac.cn/. Formal errors were different for the observations at different epoch (Li et al., 2015). Outliers were eliminated by Interquartile Range (IQR) statistic (Langbein and Bock, 2004), once outliers were found in one component, the other two components were also discarded. The epochs of whose formal errors are larger than $10 \mathrm{~mm}, 10 \mathrm{~mm}$ and 20 $\mathrm{mm}$ for East, North and Up components, were also removed, respectively (Li et al., 2018).

Here taking the SCMN station as an example, traditional EMD and weighted EMD are compared in processing details specifically. The position time 


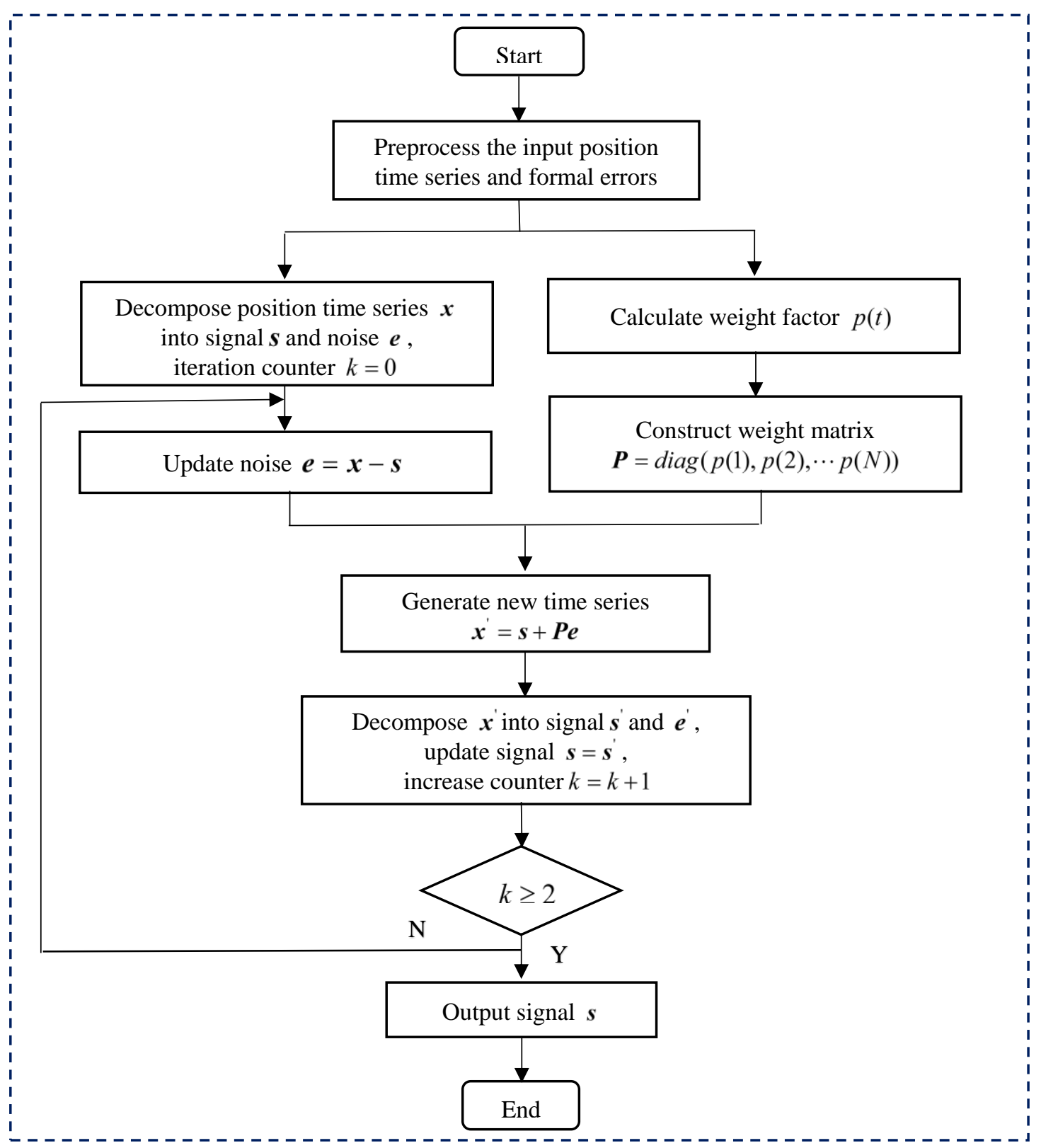

Fig. 1 The flow of weighted EMD approach.

series and formal errors of SCMN station over the period from 2011 till 2018 are presented in Figures 3 and 4, respectively. From Figure 4, it is obviously to find that the precision of time series varies from epoch to epoch. Besides, we can see that the precision of North and East components is obviously better than Up component.

EMD can decompose the GNSS position time series of SCMN station into several IMF components with frequency from high to low and one residual term (consider as IMF component). The correlation coefficient criterion is used to determine the boundary between signal and noise, and the first local minimum of correlation coefficient is taken as the boundary of IMF component. According to Equation (5), the correlation coefficients of all IMF components and original position time series are presented in Table 1 . From Table 1, we can find that the boundary between signal and noise components are all $\mathrm{IMF}_{4}$ for North, East and Up component, respectively.

According to boundary IMF component, we extract the signals from the position time series of SCMN station. The traditional EMD choose the IMF components after boundary IMF component to reconstruct signal. Traditional EMD deals with the GNSS position time series without considering formal errors, which is unreasonable. Therefore, weighted EMD calculate the weight factors of each station at different epochs by formal errors. The formal errors standard deviations of unit weight were $1.86 \mathrm{~mm}$, $1.62 \mathrm{~mm}$ and $6.20 \mathrm{~mm}$ for East, North and Up components that based on Equation (12). The 


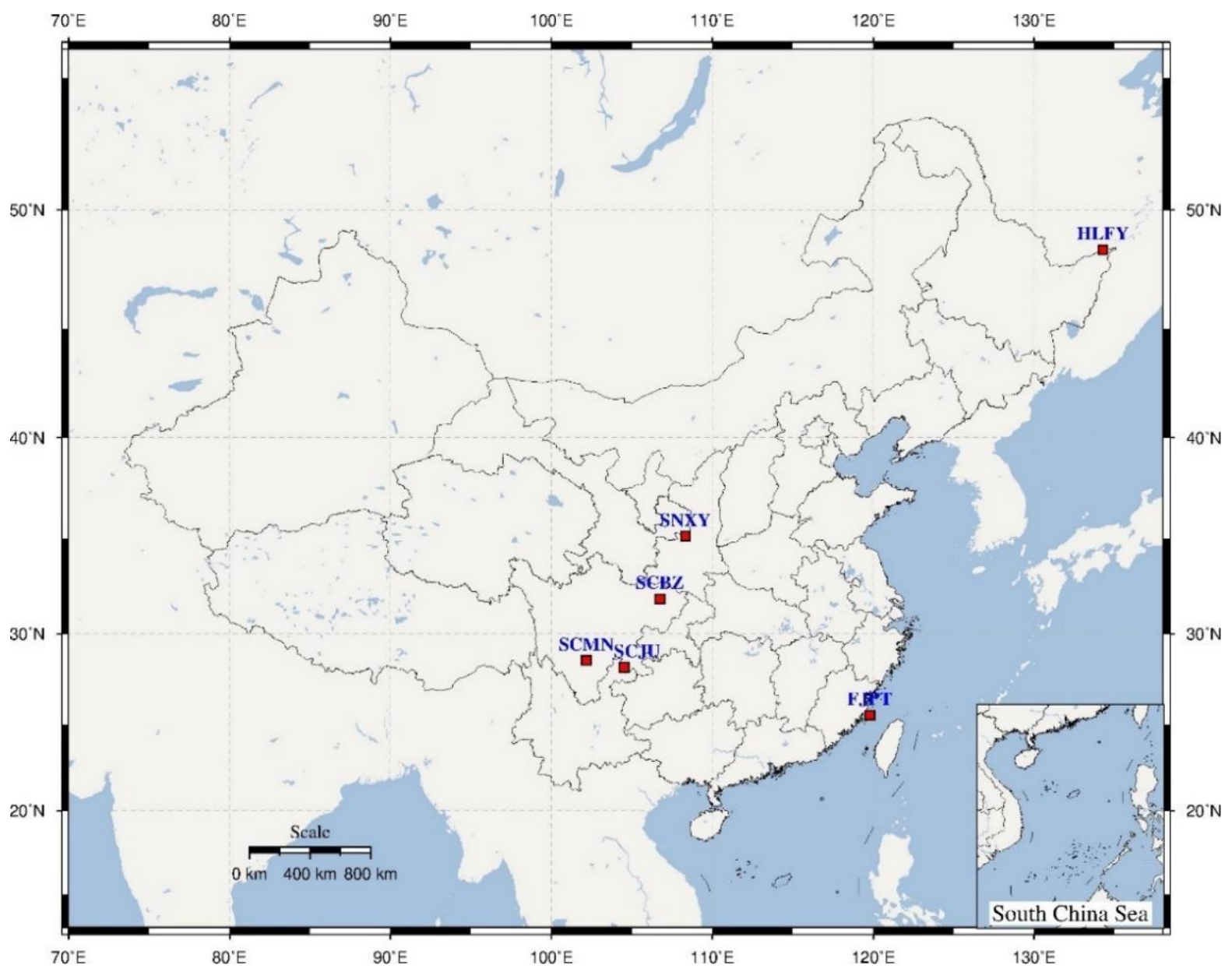

Fig. 2 Locations of the selected six stations.

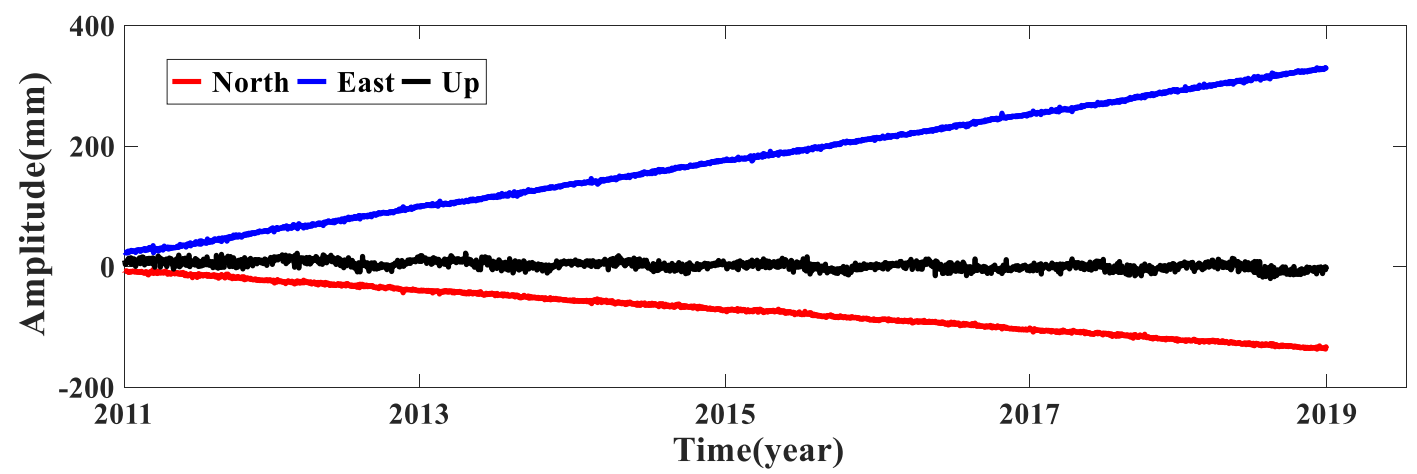

Fig. 3 Position time series of SCMN station.

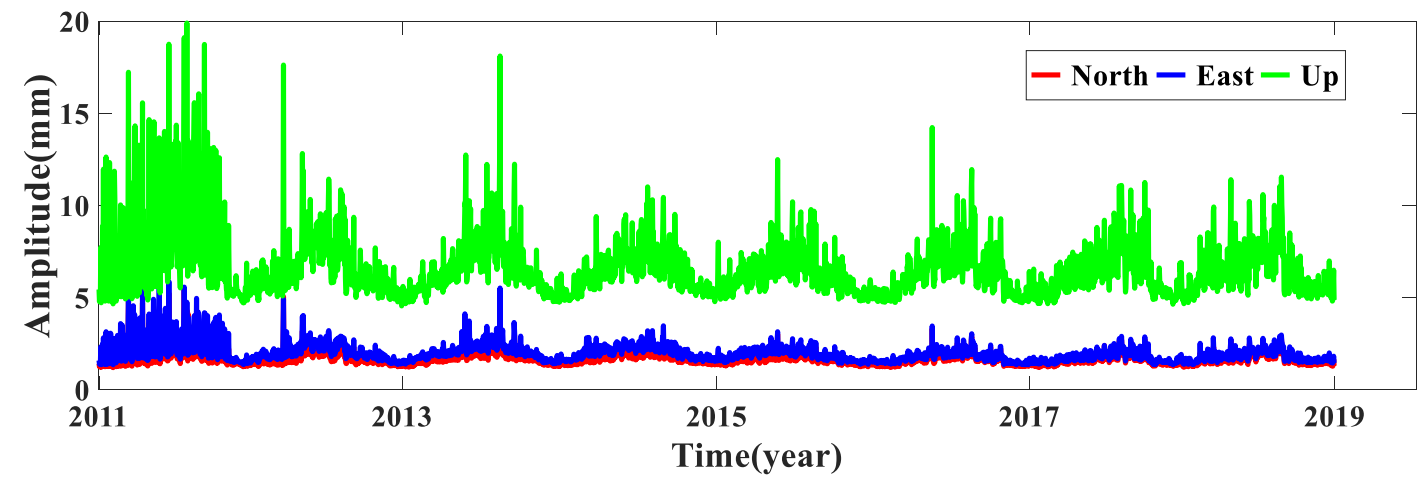

Fig. 4 Formal errors of SCMN station. 
Table 1 Correlation coefficients of all IMF components and original position time series.

\begin{tabular}{|c|c|c|c|}
\hline \multirow{2}{*}{$\mathrm{IMF}_{i}$} & \multicolumn{3}{|c|}{$R\left(\boldsymbol{x}, I M F_{i}\right)$} \\
\hline & North & East & $\mathrm{Up}$ \\
\hline $\mathrm{IMF}_{1}$ & 0.0351 & -0.0158 & 0.3959 \\
\hline $\mathrm{IMF}_{2}$ & 0.0156 & -0.0063 & 0.2837 \\
\hline $\mathrm{IMF}_{3}$ & 0.0150 & -0.0086 & 0.2006 \\
\hline $\mathrm{IMF}_{4}$ & 0.0113 & -0.0178 & 0.1591 \\
\hline $\mathrm{IMF}_{5}$ & 0.0281 & 0.0214 & 0.1733 \\
\hline $\mathrm{IMF}_{6}$ & 0.0113 & 0.0898 & 0.1156 \\
\hline $\mathrm{IMF}_{7}$ & 0.9996 & 0.9999 & 0.5334 \\
\hline $\mathrm{IMF}_{8}$ & & & 0.1130 \\
\hline $\mathrm{IMF}_{9}$ & & & 0.5883 \\
\hline
\end{tabular}

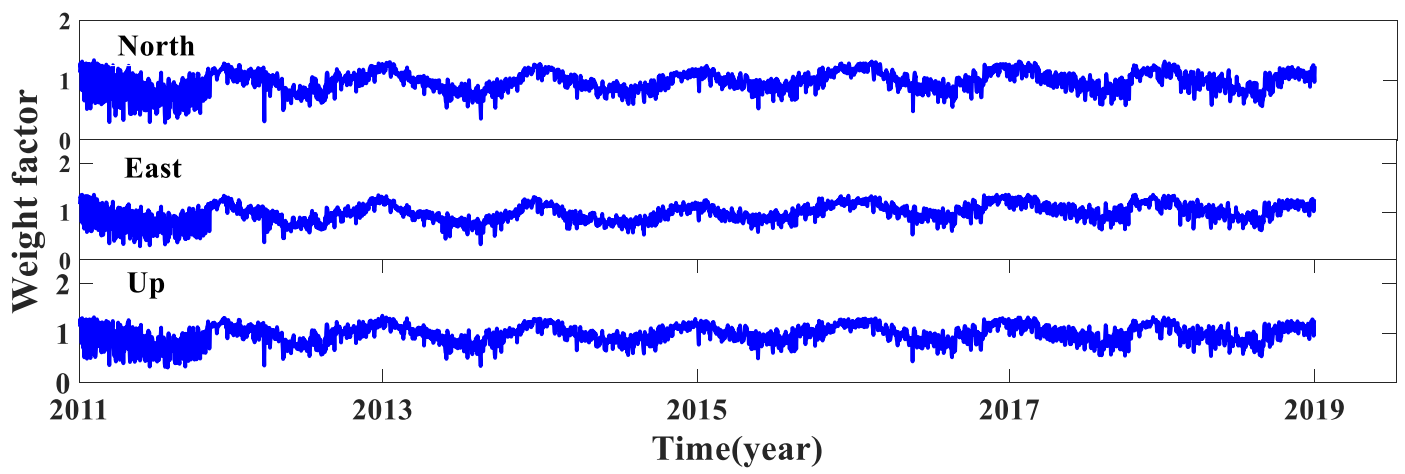

Fig. 5 Weight factors of position time series for SCMN station.

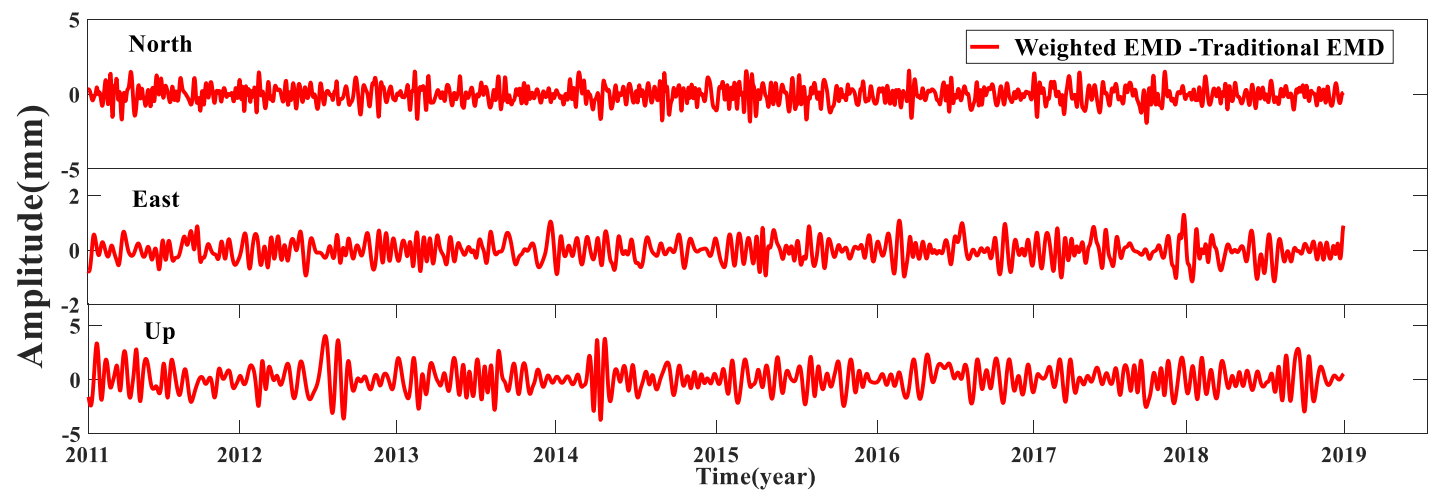

Fig. 6 The difference between weighted EMD and traditional EMD for three components.

corresponding weight factors of epochs are showed in Figure 5, weight factors varied in different epoch. The generated time series is reconstructed by Equation (8), then we analyzed and extracted signals from the generated time series by using weighted EMD. The difference of signal extraction between weighted EMD and traditional EMD with red line are presented in Figure 6. We can find that there exit some differences between the two approaches in extracting signals, with the mean absolute differences are $0.42 \mathrm{~mm}, 0.29 \mathrm{~mm}$ and $0.87 \mathrm{~mm}$ for North, East and Up components, respectively. After the signal being extracted from GNSS position time series, the fitting errors were calculated with Equation (13) for weighted
EMD and Equation (14) for traditional EMD as follows

$\hat{\sigma}_{W E M D}=\sqrt{\sum_{t=1}^{N} \hat{e}_{W E M D}^{2}(t) p(t) / N}$

$\hat{\sigma}_{E M D}=\sqrt{\sum_{t=1}^{N} \hat{e}_{E M D}^{2}(t) / N}$

where $\hat{e}_{W E M D}$ and $\hat{e}_{E M D}$ are the residual time series by weighted EMD and traditional EMD, $p$ is the weight factor for each epoch and $N$ is the length of residual time series. The fitting errors can be used to evaluate the performance of signal extraction because of the total weights are same for weighted EMD and traditional EMD. For SCMN station, the fitting errors 


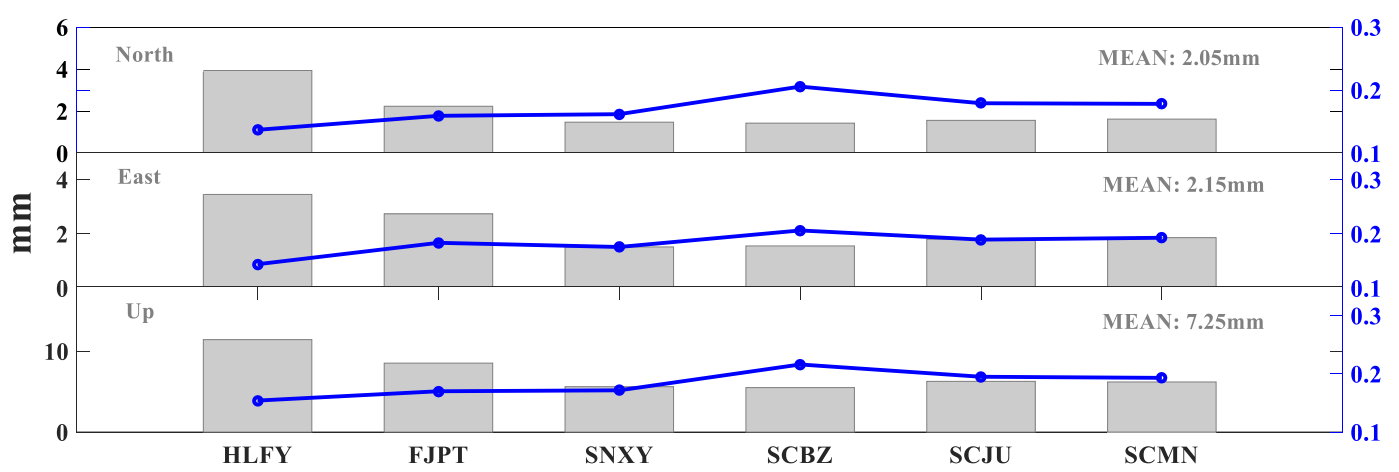

Fig. 7 The standard deviations of unit weight of formal errors (grey bar) and standard deviations of weight factors (blue line) for six stations.

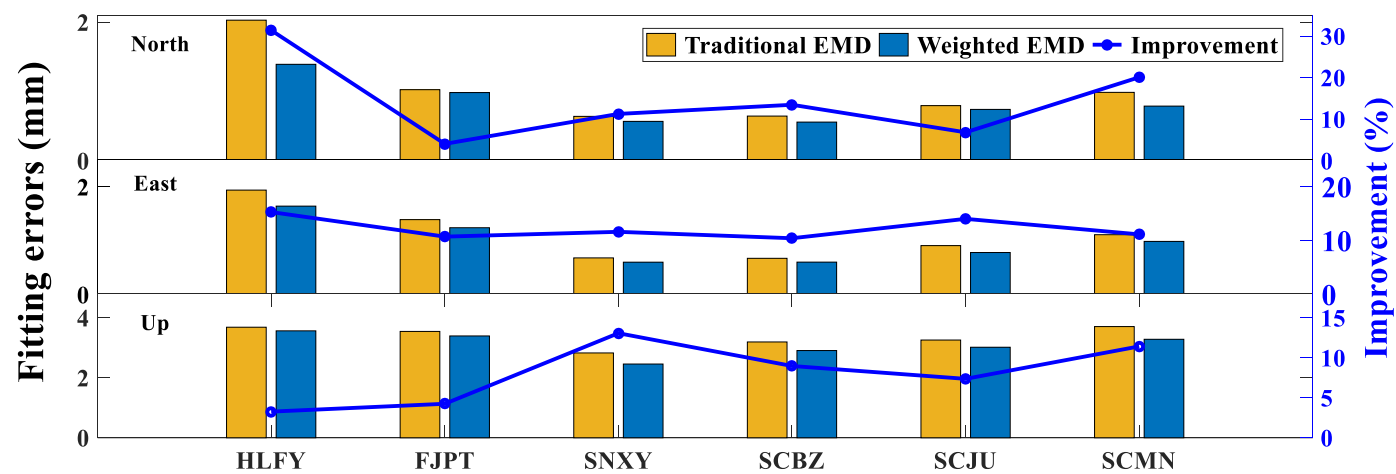

Fig. 8 Fitting errors (color bar) and relative improvements (blue line) for stations.

are $0.79 \mathrm{~mm}, 0.99 \mathrm{~mm}$ and $3.29 \mathrm{~mm}$ for weighted EMD and $0.99 \mathrm{~mm}, 1.11 \mathrm{~mm}$ and $3.71 \mathrm{~mm}$ for traditional EMD in North, East and Up components, respectively. Therefore, we can conclude that weighted EMD can extract more signals than traditional EMD.

To further testify the performance of weighted EMD with the comparison of traditional EMD, position time series of six permanent stations are adopted to analysis. The standard deviations of unit weight of formal errors derived from Equation (12) in grey bar for six stations are showed in Figure 7. We can see from Figure 7 that the corresponding standard deviations of unit weight for Up components are obviously larger than North and East components. Moreover, for the formal errors of same coordinate component, the standard deviations of unit weight are different in different stations and the mean values for six stations are $2.05 \mathrm{~mm}, 2.15 \mathrm{~mm}$ and $7.25 \mathrm{~mm}$ for North, East and Up components, respectively. Figure 7 also presents the standard deviations of weight factors with blue line, which indicated the variation of weight factors in different station. The mean standard deviations of weight factors are $0.170 \mathrm{~mm}, 0.182 \mathrm{~mm}$ and $0.183 \mathrm{~mm}$ for North, East and Up components, respectively. We can see that weight factors have the largest variation in Up component, while North component is the smallest. Additionally, both the formal errors of standard deviations of unit weights and the standard deviations of weight factors are very similar in the three components of all stations.

According to Equations (13) and (14), the fitting errors for weighted EMD and traditional EMD are calculated for six stations. Compared with traditional EMD, the relative improvement of weighted EMD is calculated as follows

$\operatorname{Im} p_{\widehat{\sigma}}=\frac{\widehat{\sigma}_{E M D}-\widehat{\sigma}_{W E M D}}{\widehat{\sigma}_{E M D}} \times 100 \%$

where $I m p_{\widehat{\sigma}}$ represents the relative improvements. The fitting errors (color bar) and the improvements of weighted EMD relative to traditional EMD (blue line) are shown in Figure 8. As we can be seen from Figure 8, the fitting errors of weighted EMD are all smaller than traditional EMD, which shows that weighted EMD performs better than traditional EMD in extracting signals from position time series. For the same station, the improvement of different components is varied. The mean improvements of weighted EMD relative to traditional EMD for six stations are $14.52 \%, 12.25 \%$ and $8.06 \%$ for North, East and Up components, respectively. Additionally, 
Table 2 The average RMSEs (mm) and Imps of weighted EMD relative to traditional EMD.

\begin{tabular}{ccccccc}
\hline \multirow{2}{*}{ Station } & \multicolumn{3}{c}{ SCMN } & & & \multicolumn{2}{c}{ BJGB } & \\
\cline { 2 - 7 } & $\mathrm{E}$ & $\mathrm{N}$ & $\mathrm{U}$ & $\mathrm{E}$ & $\mathrm{N}$ & $\mathrm{U}$ \\
\hline Traditional EMD & 0.8203 & 0.7078 & 1.8538 & 0.7790 & 0.7342 & 1.0804 \\
Weighted EMD & 0.7040 & 0.6261 & 1.7194 & 0.7434 & 0.6796 & 1.0118 \\
Imp (\%) & 14.18 & 11.54 & 7.25 & 4.57 & 7.44 & 6.35 \\
\hline \multirow{2}{*}{ Station } & & $\mathrm{SCJU}$ & & & $\mathrm{SCBZ}$ & \\
\cline { 2 - 7 } & $\mathrm{E}$ & $\mathrm{N}$ & $\mathrm{U}$ & $\mathrm{E}$ & $\mathrm{N}$ & $\mathrm{U}$ \\
\hline Traditional EMD & 0.7236 & 0.6449 & 1.3049 & 0.6994 & 0.5340 & 1.2439 \\
Weighted EMD & 0.6576 & 0.6096 & 1.1983 & 0.6403 & 0.4589 & 1.1744 \\
Imp (\%) & 9.12 & 5.47 & 8.17 & 8.45 & 14.06 & 5.59 \\
\hline
\end{tabular}

the mean improvement of horizontal component is better than vertical component and North component performs best.

\section{SYNTHETIC TIME SERIES ANALYSIS}

The simulation experiments were further carried out in this section to test and verify the performance of weighted EMD. The position time series of four permanent stations (SCMN, BJGB, SCJU, SCBZ) were randomly selected. In this section, the extracted signal by traditional EMD is regarded as true signal $\boldsymbol{s}$ and $\boldsymbol{e}$ is the normally distributed noise with zero mean and variance $\sigma^{2}(t)$. Therefore, the simulated time series $\boldsymbol{x}$ can be generated as follows

$x=s+e$

As a result of the true signal is known, the root mean square error (RMSE) of the extracted signal can be calculated as follows

$R M S E=\sqrt{\frac{\sum_{t=1}^{N}(s(t)-\hat{s}(t))^{2}}{N}}$

where, $s(t)$ and $\hat{s}(t)$ represent the true signal and the extracted signal at $t$ epoch, $N$ is the length of true signal. RMSE reflects the difference between the extracted signal and the true signal, the smaller the RMSE computed from Equation (17), the more accurate the extracted signal. The RMSE improvement of weighted EMD relative to traditional EMD is computed as follows

$I m p=\frac{R M S E_{E M D}-R M S E_{W E M D}}{R M S E_{E M D}} \times 100 \%$

where $R M S E_{E M D}$ and $R M S E_{W E M D}$ represent the RMSE of traditional EMD and weighted EMD, respectively. Imp denotes the RMSE improvement of weighted EMD relative to traditional EMD.

For the sake of obtaining statistically reliable results, the simulation experiments are repeated 100 times. The same processing strategies are adopted to deal with all simulation time series of SCBZ, BJGB, SCJU and SCMN stations as those used in Section 3. Average RMSEs are calculated for 100 simulation experiments, and Equation (18) is applied to compute the relative improvements of weighted EMD with respect to traditional EMD at the same time. From Table 2, we can see that each station has a certain degree of relative improvement for three components. The mean relative improvements of all stations are $9.08 \%, 9.63 \%$ and $6.84 \%$ for East, North and Up components, respectively. The improvement for the North component is the highest, followed by East component, and Up component performed worst, which are consistent with the results of real position time series analysis in Section 3, indicating weighted EMD performs better than traditional EMD in extracting signals from GNSS position time series.

\section{CONCLUSIONS}

Considering the formal errors are provided along with GNSS position time series available, traditional EMD did not make full use of the formal errors. Weighted EMD is proposed in this paper for extracting signals from noisy GNSS position time series, in which formal errors are applied to construct weight factors. The traditional EMD and weighted EMD were adopted to process the real GNSS position time series of six permanent stations from 2011 to 2018. The results show that weighted EMD performs better than traditional EMD in filtering noise and extracting signals. The fitting errors of weighted EMD are smaller than those of traditional EMD for all six stations, which means that weighted EMD can extract more signals. The corresponding improvements of weighted EMD relative to traditional EMD are $14.52 \%, 12.25 \%$ and $8.06 \%$ for North, East and Up components, respectively. Among three components, the corresponding improvement of North component is the highest, East component rank second, Up component at last. Furthermore, the corresponding improvement of horizontal component is better than vertical component. To further validate the advantage of weighted EMD relative to traditional EMD, 100 simulation experiments were performed using four stations separately that based on simulated time series. The results show that the signal extracted by weighted EMD was closer to true signal in comparison with 
traditional EMD. The improvements of mean RMSEs are $9.62 \%, 9.08 \%$ and $6.84 \%$ for North, East and Up components, respectively. Overall, both real and synthetic experiments highlight the effectiveness of weighted EMD in signal extraction. Therefore, formal errors should be considered when using EMD to extract signal from GNSS position time series.

\section{ACKNOWLEDGMENTS}

This work is mainly funded by Science and Technology Project of the Education Department of Jiangxi Province (209507) and the Natural Science Foundation of China (42064001). We acknowledge the CMONOC for providing raw position time series calculated with GAMIT (http://www.cgps.ac.cn/). We also acknowledge the careful and constructive reviews of Editor and two anonymous reviewers, who have helped improve the manuscript.

\section{REFERENCES}

Bogusz, J. and Klos, A.: 2016, On the significance of periodic signals in noise analysis of GPS station coordinates time series. GPS Solut., 20, 4, 655-664. DOI: 10.1007/s10291-015-0478-9

Chen, B.Z., Bian, J.W., Ding, K.H., Wu, H.C. and Li, H.W.: 2020, Extracting seasonal signals in GNSS coordinate time series via weighted nuclear norm minimization. Remote Sens., 12, 12, 2027. DOI: $10.3390 /$ rs 12122027

Dai, H.L., Sun, F.P., Xiao, K., Zhu, X.H. and Liu, J.: 2021, Application of wavelet decomposition and singular spectrum analysis in GNSS station coordinate time series. Geomat. Inf. Sci. Wuhan Univ., 46, 3, 371-380, (in Chinese). DOI: 10.13203/j.whugis20190107

Dong, D., Fang, P., Bock, F, Webb, F., Prawirondirdjo, L., Kedar, S. and Jamason, P.: 2006, Spatiotemporal filtering using principal component analysis and Karhunen-Loeve expansion approaches for regional GPS network analysis. J. Geophys. Res., 111, B3, B03405. DOI: 10.1029/2005JB003806

Golyandina, N. and Zhigljavsky, A.: 2013, Singular spectrum analysis for time series. Springer Berlin Heidelberg. DOI: 10.1007/978-3-642-34913-3

Huang, Y., Tian, L.Y., Bai, Y., Zhang, Z.Q. and Zhang, F.P.: 2014, GNSS coordinates time series noise characteristics analysis. GNSS World of China, 39, 4, 16-20+25, (in Chinese). DOI: 10.13442/j.gnss.10089268.2014.04.005

Huang, N.E., Long, S.R., Wu, M.L.C., Shih, H.H., Zheng, Q.N., Yen, N.C., Tung, C.C., Liu, H.H. and Shen, Z.: 1998, The empirical mode decomposition and the Hilbert spectrum for nonlinear and non-stationary time series analysis. Proc. Royal Society A: Mathematical physical and engineering sciences, 454, 1971, $903-$ 995. DOI: 10.1098/rspa.1998.0193

Ji, K.P., Shen, Y.Z. and Wang, F.W: 2020, Signal extraction from GNSS position time series using weighted wavelet analysis. Remote Sens., 12, 6, 992. DOI: $10.3390 / \mathrm{rs} 12060992$

Langbein, J. and Bock, Y.: 2004, High-rate real-time GPS network at Parkfield: Utility for detecting fault slip and seismic displacements. Geophys. Res. Lett., 31, 15. DOI: 10.1029/2003GL019408

Lei, D. and Zhong, S.S.: 2013, Aircraft engine health signal denoising based on singular value decomposition and empirical mode decomposition methods. J. Jilin Univ. Eng. Technol., 43, 3, 764-770. DOI: $10.7964 /$ jdxbgxb201303034

Li, S.Y., Shen, W.B., Pan, Y.J. and Zhang, T.X.: 2020, Surface seasonal mass changes and vertical crustal deformation in North China from GPS and GRACE measurements. Geod. Geodyn., 11, 1, 46-55. DOI: 10.1016/j.geog.2019.05.002

Li, W.W., Shen, Y.Z. and Li, B.F.: 2015, Weighted spatiotemporal filtering using principal component analysis for analyzing regional GNSS position time series. Acta Geod. Geophys., 50, 4, 419-436. DOI: $10.1007 / \mathrm{s} 40328-015-0100-1$

Li, W.W. and Shen, Y.Z.: 2018, The consideration of formal errors in spatiotemporal filtering using principal component analysis for regional GNSS position time series. Remote Sens., 10, 4, 534.

DOI: $10.3390 / \mathrm{rs} 10040534$

Li, Z., Jiang, W.P., Liu, H.F. and Qu, X.C.: 2012, Noise model establishment and analysis of IGS reference station coordinate time series inside China. Acta Geod. Cartogr. Sin., 41, 4, 496-503, (in Chinese).

Liu, S.D. and Chen, Z.X.: 2019, Nonlinear vibration signal denoising based on singular value decomposition and EEMD. J. Detect. Control., 4, 3, 37-42, (in Chinese).

Ma, J., Cao, C.D., Min, Y. and Zhou, L.: 2020, Removal of colored noise in GNSS station coordinate time series based on principal component analysis. Academic Exchange Center of China Satellite Navigation System Management Office. Proc. 11th China Satellite Navigation Annual Conference -S05.

Ma, J., Cao, C.D., Jiang, W.P. and Zhou, L.: 2020, Method for removing colored noise of GNSS station coordinate time series based on wavelet packet coefficient information entropy. Geomat. Inf. Sci. Wuhan Univ., (in Chinese). DOI: $10.13203 /$ j.whugis 20190353

Ma, J.: 2019, Establishment of strict three dimensional noise model for GPS coordinate time series. Acta Geod. Cartogr. Sin., 48, 9, 1205. DOI: 10.11947/j.AGCS.2019.20180584

Ming, F., Yang, Y.X., Zeng, A.M. and Jing, Y.F.: 2016, Analysis of seasonal signals and long-term trends in the height time series of IGS sites in China. Sci. China Earth Sci., 59, 6, 1283-1291.

DOI: $10.1007 / \mathrm{s} 11430-016-5285-9$

Nikolaidis, R.: 2002, Observation of geodetic and seismic deformation with the Global Positioning System. Ph.D Thesis, University of California, San Diego.

Peltier, W.R., Argus, D.F. and Drummond, R.: 2015, Space geodesy constrains ice age terminal deglaciation: The global ICE-6G-C (VM5a) model. J. Geophys. Res., Solid Earth, 120, 1, 450-487. DOI: $10.1002 / 2014 J B 011176$

Qian, C., Liu, H., Ding, Z.G. and Zheng, H.Y.: 2015, Longterm stability of reference stations by taking nontectonic deformation into account. Geomat. Inf. Sci. Wuhan Univ., 40, 9, 1259-1265, (in Chinese). DOI: 10.13203/j.whugis20130715 
Shen, Y. Z., Wang, F.W., Li, W.W. and Chen, Q.J.: 2018, Singular spectrum analysis for heterogeneous time series by taking its formal errors into account. Acta Geodyn. Geomater., 15, 4, 395-403.

DOI: 10.13168/AGG.2018.0029

Song, J.X., Lei, Q., Fang, X., Zhang, W.P. and Cao, W.: 2017, Filtering method based on wavelet transform of armored targets sound signal. J. Detect. Control, 39, 4, 61-66, (in Chinese).

Tobita, M.: 2016, Combined logarithmic and exponential function model for fitting postseismic GNSS time series after 2011 Tohoku-Oki earthquake. Earth Planets Sp., 68, 1, 41.

DOI: $10.1186 / \mathrm{s} 40623-016-0422-4$

Tufts, D.W., Kumaresan, R. and Kirsteins, I.: 1982, Data adaptive signal estimation by singular value decomposition of a data matrix. Proc. IEEE, 70, 6, 684-685.

DOI: 10.1109/PROC.1982.12367

Wang, F.W., Shen, Y.Z., Li, W.W. and Chen Q.J.: 2018, Singular spectrum analysis for heterogeneous time series by taking its formal errors into account. Acta Geodyn. Geomater., 15, 4, 329-403.

DOI: 10.13168/AGG.2018.0029

Wei, G.J., Chen, C., Huang, Y.Y. and Wei, H.L.: 2019, Research on periodic analysis and noise reduction method of elevation coordinate timing of CORS based on wavelet transform. GNSS World of China, 44, 4, 61-67, (in Chinese).

DOI: 10.13442/j.gnss.1008-9268.2019.04.009

Zhang, S.C., Li, Z.Y., He, Y.F., Hou, X.W., He, Z.X. and Wang, Q.Y.: 2018, Extracting of periodic component of GNSS vertical time series using EMD. Sci. Surv. Mapp., 43, 8, 80-84+96, (in Chinese). DOI: $10.16251 /$ j.cnki.1009-2307.2018.08.013

Zhu, Z.H., Zhou, X.H., Deng, L.S., Wang, K.H. and Zhou, B.Y.: 2017, Quantitative analysis of geophysical sources of common mode component in CMONOC GPS coordinate time series. Adv. Sp. Res., 60, 12, 2896-2909. DOI: 10.1016/j.asr.2017.05.002 